\title{
A Novel Way to Construct the Fibonacci Sequence and the Uni-Phi-cation of Mathematics and Physics
}

\author{
David F. Haight \\ Department of History and Philosophy, Plymouth State University, Plymouth, New Hampshire, USA
}

\section{Email address:}

dhaight@plymouth.edu

\section{To cite this article:}

David F. Haight. A Novel Way to Construct the Fibonacci Sequence and the Uni-Phi-cation of Mathematics and Physics. Pure and Applied Mathematics Journal. Vol. 4, No. 4, 2015, pp. 139-146. doi: 10.11648/j.pamj.20150404.11

\begin{abstract}
When the Fibonacci number sequence is based on the number seven and its multiples, the Fibonacci sequence self-reflexively reappears when differences are calculated between it and this new number-seven-based Fibonacci sequence. The same thing happens with Lucas numbers. Can this same procedure be applied to any two numbers at the beginning of a Fibonacci/Lucas-like sequence? The answer is in the negative. This special quality of the golden proportion casts light on the fine structure constant of hydrogen, which is the unique, lightest, and most pervasive element in nature, plus other constants in nature, all of which have a dimensionless number close to the golden proportion (Phi) of the Fibonacci sequence, and provides the basis for the binary computer code as well as a uni-Phi-ed theory of mathematics and physics.
\end{abstract}

Keywords: Fibonacci Sequence, Lucas Sequence, Golden Proportion, "Prime” Prime Number, Hydrogen Fine Structure, The Physical Constants, Binary Computer Code, A Uni-Phi-ed Theory of Mathematics

\section{Introduction: One, Two, Three, One, Two, Three}

Let us begin with ten already established theorems and some related theorems regarding the perennial golden proportion:

1. The golden division is the only one in which the three unequal "divisions"--the small part, the large part, and the whole, all working together simultaneously--remain united even in their separation (the "three that are two that are one"). This proportion is very clever: it cleaves itself and then cleaves to itself.

2. This division is also unique, in a class by itself, unlike any other, because it is the only section created out of the self-interaction of the number one (the simplest continued fraction and the simplest nested or "radical" radical).

3. This division is the only two-term proportion (the "one that is two"). All others have at least three.

4. The Fibonacci sequence of numbers, through collection and division, fusion and fission, integration and differentiation, as in calculus, converges to Phi, the golden proportion.

5. The golden proportion is the only one in which its (legato) addition and (stoccato) multiplication of itself are equivalent. It is both an arithmetic and a geometrical progression, two sides of the same coin (another "two that are one"), and is the basis of logarithms and exponentials (logarithms transform multiplication into addition, and exponentials transform addition into multiplication).

6. Similar to Phi, the number six is the only number that is both the addition and multiplication of its divisors $(1,2$, 3)--the most "perfect" perfect number.

7. The number three is the only number that is equal to all the numbers that precede it $(1+2)$--another "three-in-one."

8. All duals (two numbers) converge to the non-dualistic Phi just as all major and minor musical scales, dominants and subdominants, regress to the mean.

9. Phi is the one and only solution to the equation $\mathrm{x}-(1 / \mathrm{x})=$ 1. In other words, Phi is self-reciprocating. This curious self-reciprocity is also present in Phi's relationship with $i$, the square root of minus one: $(i \phi)+1 /(i \phi)=i$.

10. Phi is the one and only number that is one less than its square.

Does anything follow from these statements that has not already been found after all these many hundreds of years? What more can the "science and art of combination" disclose?

\section{Thesis and Proof}

An answer lies within the very first numbers of the 
Fibonacci sequence, $0,1,2,3$ when we add them together, along with their unity (1), to obtain the number 7 . There is justification in making this claim since the number three, like Phi, is actually three-in-one and is structurally self-supporting like a triangle-truss (torus). Three is implicit in unity, and a triangle is the simplest polygon. Moreover, the number six is the only number that, like Phi, is both the addition and multiplication of its divisors. When six and one are added together the result is the number 7 , which is yet another self-derived number that is not divisible by any other number than itself and one.

Following the same progression as the Fibonacci sequence, the resulting sequence of numbers looks like this: 3 -in- 1 is 1,1 $+2+3+1=7,1+2+3+1+7=14,7+14=21,14+21=$ $35,21+35=56,35+56=91,56+91=147,91+147=238$, $147+238=385,238+385=623,385+623=1008,623+$ $1008=1631$, and so on. Did the reader notice that this sequence based on multiples of the number seven follows exactly the Fibonacci sequence itself: 0, 1, 1 (7), 2 (14), 3 (21), 5 (35), 8 (56), 13 (91), 21 (147), 34 (238), 55 (385), 89 (623), 144 (1008), 233 (1631), and so on? If we begin the sequence with something like Phi, namely, the unity of the first three numbers, the unfolding sequence becomes the Fibonacci sequence that converges to Phi.

This same sequential unfolding surprisingly reappears whenever we compare and contrast the "old" Fibonacci sequence with this "new" (novel) one:

\begin{tabular}{|c|c|c|}
\hline Old Sequence & New Sequence & Differences \\
\hline 0 & 0 & 0 \\
\hline 1 & 1 & 0 \\
\hline 1 & 1 & 0 \\
\hline 2 & 2 & 0 \\
\hline 3 & 3 & 0 \\
\hline 5 & 6 & +1 \\
\hline 8 & 7 & -1 \\
\hline 13 & 14 & +1 \\
\hline 21 & 21 & 0 \\
\hline 34 & 35 & +1 \\
\hline 55 & 56 & +1 \\
\hline 89 & 91 & +2 \\
\hline 144 & 147 & +3 \\
\hline 233 & 238 & +5 \\
\hline 377 & 385 & +8 \\
\hline 610 & 623 & +13 \\
\hline 987 & 1008 & +21 \\
\hline 1597 & 1631 & +34 \\
\hline 2584 & 2639 & +55 \\
\hline 4181 & 4270 & +89 \\
\hline 6765 & 6909 & +144 \\
\hline 10946 & 11179 & +233 \\
\hline 17711 & 18088 & +377 \\
\hline
\end{tabular}

Etc.
As it turns out, the difference between the two Fibonacci sequences makes no difference, which is completely consistent with Phi since it is itself a unity-in-difference.

Is this procedure also true of Lucas numbers? Let's see:

$\begin{array}{ccc}\text { Lucas } & \text { Fibonacci } & \text { Differences } \\ 0 & 0 & 0 \\ 2 & 1 & +1 \\ 1 & 1 & 0 \\ 3 & 2 & +1 \\ 4 & 3 & +1 \\ 7 & 5 & +2 \\ 11 & 8 & +3 \\ 18 & 13 & +5 \\ 29 & 21 & +8 \\ 47 & 34 & +13 \\ 76 & 55 & +21\end{array}$

Etc.

Indeed it is. We could also begin the Lucas sequence with just 1, 3, 4, etc., and obtain the same differences; it doesn't matter. (In passing it can be mentioned that 1, 3 and 7 are also Lucas numbers.)

The (inevitable) question is, does this happen with any set of two numbers at the beginning? And the answer is that it doesn't. (Let the reader choose any other pair and see for him/herself.) The Fibonacci progression only appears if the sequence begins with a variation of the three that are one.

\section{Clocking the Infinite Prime Numbers, or Prime Time: Further Results}

Something similar happens, however, with respect to the generation of prime numbers in what might be called the prime number digital clock where primes line up in spider-leg-like fashion only on the eight rays around a spider-web "24-hour" period, using Gottfried Wilhelm Leibniz's formula or weave-web-wave function $6 \mathrm{n}$ plus or minus 1 where $\mathrm{n}$ is a whole number. After the initial numbers 1,2 , and 3, all primes (and admittedly some composites) mathematically follow from Leibniz's formula: 5 and 7 are 6 $\mathrm{x} 1$ minus and plus 1,11 and 13 are $6 \times 2$ minus and plus 1, 17 and 19 are $6 \times 3$ minus and plus 1 , and then 23 is $6 \times 4$ minus 1 , with 25 or 1 beginning a new cycle in another 24 -hour elliptic modular period. Here again we find the unity of 1,2, and 3 in 6 that began our "new" Fibonacci sequence.

Of the 24 diameters on the prime number clock, three separate "eights" appear: eight rays unfold the primes divisible by one (!), eight rays unfold the numbers divisible by two (!), and eight rays unfold numbers divisible by three (!). In true Pythagorean fashion all squares of primes respectively queue up on just the first ray of one squared--25, $49,121,169$, etc.--because the number one is added to multiples of 24. "There is geometry in the humming of the primes, there is music in the spacing of the primes." The 
primes dance to the rhythm of this "Pied Piper" choreography and are therefore ratified (rated or rationed).

It is also possible to think of this modular clock as having a pendulum that traces a cycloid arc back and forth according to the law of quickest descent or least time. This pendulum beats out the winding number that provides the harmonic spacing of the modes in the modular clock of primes. (For more explanation of the synchronized symphonic structure of the primes, see my papers, "Summa Characteristica and the Riemann Hypothesis: Scaling Riemann's Mountain," vol. 11, no. 6, December 2008, and "Generalizing Riemann: from the L-Functions to the Birch/Swinnerton-Dyer Conjecture," vol. 13, no. 5, October 2010, in the Journal of Interdisciplinary Mathematics, both papers being re-printed [e-printed] electronically online by Taylor and Francis Publishing Group, 2013.)

Prime numbers, like natural elements, are periodic, as the prime number clock shows. (Like the flat periodic table, which is an unfolded torus donut cut both laterally and longitudinally, the prime number clock is a vortex ring that contains a special octave periodicity. The torus is a tor, tower, or truss like the self-supporting triangle.) The question then naturally arises, is there a "prime" prime number hidden in this periodicity just as there is in the periodic table of natural elements?

In the periodic table there are six (!) noble gases, plus the "King" (Arc-tor or Arthur) element hydrogen, making a total of seven (!) "notes" in this octave period. And all the natural elements are literally spin-offs from hydrogen, the lightest and most prevalent element in the universe. As is widely known, hydrogen has the fine structure constant of $137+$ and also has electrons whose probable histories follow the Fibonacci number sequence that converges to the golden angle number of 137.5 degrees (see physicist H. E. Huntley's book, The Divine Proportion, page 156). Since the ideal divergence angle in nature is the golden proportion of 137.5 degrees, could this unique proportion be the reason why 137 is the "prime" prime number or paradigm prime in nature, beginning with hydrogen? Is this the prime time(r) in a prime paradigm?

There is considerable evidence that the golden proportion is the foundation for the unification of mathematics and physics. Not only do any two numbers whatsoever when collected and divided in the usual Fibonacci manner taper off toward Phi, like the winding down of a pendulum clock or dampened physical sine wave function, but all of the fundamental operations and operators appear in what physicist Richard Feynman referred to as "the most remarkable formula in mathematics," Leonhard Euler's identity, $e^{\pi i}+1=0$ (which upon being seen makes one positively "Pi-eyed"). When Phi is inserted into it, where Phi $=$ the square root of 5 minus $e^{\pi i}$, Leonhard Euler's formula, $-e$ to the power of Pi times $i$, is equal to 1. (By the way, the natural $\log e=1.37^{\mathrm{Pi}}, \mathrm{Phi}^{1.37}=\mathrm{Pi} / \mathrm{Phi}$, and $13.7^{1.37} / \mathrm{Phi}^{2}=$ 13.7.)

As Feynman himself discovered, $e^{\pi i}=$ one divided by Phi minus Phi. And, as Leibniz showed, all numbers can be translated into the binary scale of zeros and ones, and (what Leibniz did not know) the binary code is based on Phi's radical roots (the simplest nested radical) that oscillate back and forth between zero and one. Strangely but beautifully, the square of any Fibonacci number differs from the multiplication of its immediate predecessor and successor by only one, in another plus and minus one alternation, e.g., $21^{2}$ $=441,13 \times 34=442 ; 89^{2}=7921,55 \times 144=7920$; etc.

Leibniz rightly claimed "that all lines of motion in the whole of geometry are reduced to two motions only, one in a straight line and the other in a circle" ("The Great Art of Thinking," in Leibniz's Philosophical Writings, ed., G. H. R. Parkinson, page 3), the same as all numbers being compressible to zeros and ones. Significantly, the line and the circle are the limiting cases of the golden Phi logarithmic spiral. A line is the minimum possible area, and a circle is the maximum possible area; all other polygons are intermediate between these two figures and are combinations of them.

Unfortunately, what Leibniz did not discover in both cases is that Phi is the unity-in-diversity which bridges the gap between these binary opposites. A one-dimensional straight line gets moved into a two-dimensional circle because of the joint arithmetic and geometric character of non-dual Phi. The (golden) intersection between dimensions is the result of the unique self-intersection of the dimensionless golden section where infinity comes to rest. (In other words, this is as far down as the Fibonacci rabbit hole goes.)

Leibniz's law of continuity between dimensions (or derivatives in calculus) and the limit with the unlimited depends upon Phi, since by multiplication and addition to a line a plane is produced; by multiplication and addition to a plane a solid is produced; by multiplication and addition to a solid a hypersolid is produced; etc. In the golden logarithmic spiral the intervals add up while the ratio of frequencies multiply; while the radial angle adds up in an arithmetic progression, the spiral radius grows up in a geometric expansion. Together these two progressions produce the harmonic progression of all the subsequently derived dimensions. (This spiral is the mill that grinds the grist of a lower dimension into a higher, more refined one.)

These extrapolated derivations come right out of Descartes's equiangular spiral (rainbow) of analytical geometry, the inspiring (spiral) forerunner of Leibniz's and Newton's calculus. Phi is the only proportion that remains the same or true to itself by both adding and multiplying itself (as a golden spiral, its state and rate of growth are the same [ratification]), which makes it the portal from one dimension to another and the common boundary or coupling constant between linear and non-linear expansion, order and chaos, the limit and the unlimited. In a word, Phi is peerless.

Whether he knew it or not, Leibniz's translation of the decimal code into the binary system also shows a deep connection between unity (ones) and the progression of 1, 3, 7 (unitriseptium) that consistently appears whenever the block (.142857) and period (132645) of the number seven (take note!) is applied: 


\begin{tabular}{|c|c|c|c|}
\hline Decimal & Binary & Decimal & Binary \\
\hline $1=2^{0}=1$ & $=0 \times 7+1$ & 17 & $=10001$ \\
\hline $2=2^{1}=10$ & $=1 \times 7+3$ & 18 & $=10010$ \\
\hline 3 & $=11$ & 19 & $=10011$ \\
\hline $4=2^{2}=100$ & $=14 \times 7+2$ & 20 & $=10100$ \\
\hline 5 & $=101$ & 21 & $=10101$ \\
\hline 6 & $=110$ & 22 & $=10110$ \\
\hline 7 & $=111$ & 23 & $=10111$ \\
\hline $8=2^{3}=1000$ & $=142 \times 7+6$ & 24 & $=11000$ \\
\hline 9 & $=1001$ & 25 & $=11001$ \\
\hline 10 & $=1010$ & 26 & $=11010$ \\
\hline 11 & $=1011$ & 27 & $=11011$ \\
\hline 12 & $=1100$ & 28 & $=11100$ \\
\hline 13 & $=1101$ & 29 & $=11101$ \\
\hline 14 & $=1110$ & 30 & $=11110$ \\
\hline 15 & $=1111$ & 31 & $=11111$ \\
\hline $16=2^{4}=10000$ & $=1428 \times 7+4$ & $32=2^{5}=100000$ & $=14285 \times 7+5$ \\
\hline
\end{tabular}

(Does the reader see the 1, 3, 7 progression? If not, count the number of numbers in the far left column between 10 and 100 , then between 100 and 1000, and then between 1000 and 10000 , respectively. In other words, count the numbers in between all of the binary numbers that have only ones in them. This 1, 3, 7 progression provides us with a deep clue as to the unity [ones] of the binary number code.)

Here are the block and period of the prime number seven:

$\begin{array}{cc}\text { Block } & \text { Period } \\ 3 \times 7 & =21 \\ 2 \times 7 & =14 \\ 6 \times 7 & =42 \\ 4 \times 7 & =28 \\ 5 \times 7 & =35 \\ 1 \times 7 & =7\end{array}$

This astonishing result comes from the fact that $1 / 7$ (unity divided by seven) produces the block of 326451 and the octave-like doubling period of .14-28-57. Seven is the smallest number that when divided into unity makes a cycle or period of recurring digits. Multiplication of 142857 by other numbers also results in a cycle of the same numbers. This octave progression of .142857 is the binary/decimal symphony of all the numbers based upon a single note (137+). Using his discovery of the binary code, Leibniz ingeniously built the first mechanical computer that can add, subtract, multiply, divide, and do both extraction and squaring of roots. (Although it preceded Leibniz's model, Blaise Pascal's computer could only add and subtract.) It is very hard to imagine what today's world would be like without this revolutionary combination of both pure and applied mathematics.

In sum, all numbers can be reduced to sums of the octave-doubling powers of two $\left(2^{0}=1,2^{1}=2,2^{2}=4,2^{3}=8,2^{4}\right.$ $=16$, etc.) and with the help of the number seven and its cycles all digital numbers compress to a unity of ones through the block and period of the number seven and the Phi progression of $1,3,7$, the summa characteristica. One number is all--all numbers in one. What Leibniz did know for sure is that this same progression appears whenever the simple binary formula, $2^{\mathrm{n}}-1$, is solved: $2^{0}-1=0,2^{1}-1=1,2^{2}-1=3,2^{3}-1=7$, etc. This formula is, for Leibniz, one of the reasons why there is something rather than nothing; it is rather like the law of least action--how easily something comes from nothing--that Leibniz discovered. The shortest distance between nothing and something (zero and one), which are the first two numbers of the Fibonacci sequence, is the least amount of action (later on in the twentieth century to become energy times time = Max Planck's constant $h$, or $137+$, in quantum mechanics).

In passing, this table of decimal/binary numbers affords us the opportunity to note that much of the mysterious (prime) number 137 in mathematics and science goes all the way back to Pythagorean "perfect" numbers that are functions of the binary number two. Each perfect power of two has an "imperfect" sum of divisors one less than the power of two itself:

$$
\begin{array}{lll}
2^{0}=0 & \text { divisors: } 0 & \text { sum of divisors }=0 \\
2^{1}=2 & \text { divisors: } 1 & \text { sum of divisors }=1 \\
2^{2}=4 & \text { divisors: } 1,2 & \text { sum of divisors }=3 \\
2^{3}=8 & \text { divisors: } 1,2,4 & \text { sum of divisors }=7
\end{array}
$$

Later on, Euclid added to this that when a power of two and the next power of two minus one are multiplied they always equal a Pythagorean perfect number:

$$
\begin{gathered}
2^{1} \times\left(2^{2}-1\right)=6 \\
2^{2} \times\left(2^{3}-1\right)=28 \\
2^{3} \times\left(2^{4}-1\right)=496 \\
2^{4} \times\left(2^{5}-1\right)=8,128
\end{gathered}
$$

(Incidentally, the number two appears more often in mathematics and physics than any other number.)

There is a further connection between $2 n-1$ and prime numbers: if and only if $2 n-1$ divides the $(n-1)$ th term of a series, then $2 n-1$ is prime; otherwise, it is composite. Euler showed that if $2 n-1$ is prime, all even perfect numbers have the form $2 n(2 n+1-1)$. The Mersenne sequence of primes, according to $\mathrm{Mp}=2 \mathrm{p}-1$, also produces the progression of 1 $3-7$.

\section{Why 137: Nature's Ratification}

"Light $[137+]$, like everything in the universe, follows the path of the beautiful," said Richard Feynman's high school physics teacher, who was quoting an anonymous source.

"The number 137 is the dominating factor of all natural phenomena," claimed physicist Max Born.

Coincidentally, this very fundamental formula $2^{\mathrm{n}}-1$ was Srinivasa Ramanujan's favorite, and, of course, $137+$ is the dimensionless number (ratio) not only of hydrogen but of 
Einstein's constant of light, Planck's constant of action and Feynman's constant charge of the electron, according to the quantum mechanical formula, $h c / 2 \mathrm{pi}^{2}=137+$. (This number is known as alpha $(\alpha)$, the first letter of the Greek alphabet, which is also the letter assigned in mathematics to the golden ratio phi or $.618 \ldots$, the reciprocal of Phi or $1.618 \ldots$.. . Because this $137+$ number is common to Newton's macroscopic constant of gravity, Einstein's macroscopic constant of light and Planck's microscopic constant of stationary action, it is a promising clue to the missing link between light, gravity, and action, or relativity theory and quantum theory, in a quantum theory of gravity.

Moreover, almost incredibly, the number one is equal to Newton's constant of gravity, Boltzmann's constant of temperature in thermodynamics, Coulomb's constant force of the atom, Einstein's constant of light, and Planck's constant of action whenever Max Planck's natural units are used to take the measurements of mass (M), length (L), and time (T). All of these five fundamental constants in nature equal the number one, using these natural units, because the elementary charge of the electron or proton equals the square root of $137+$ (see The Road to Reality, by Roger Penrose, pp. 715, 716). This equivalence amongst such a diversity of constants is unique to this ("Goldilocks") number alone. This special dimensionless number (ratio) is the only way in which all of these constants can be combined into a unity, which goes a long ways toward explaining "the unreasonable effectiveness of mathematics in relation to the physical sciences" (physicist Eugene Wigner, in his paper of the same title, in Communications on Pure and Applied mathematics 13, 1, 1960).

The dimensionless number $137+$ or ML2T, the conservation of action, is the symmetry of symmetries or the (Emmy Noether) law of all laws governing the conservation laws of the universe. "There is a deep connection between the symmetry principles and the conservation laws, but that connection requires that the minimum principle (of least action) be assumed.... In the last analysis the connection of symmetry laws to conservation laws comes from quantum mechanics [namely, Planck's constant of least action 137+]," wrote Richard Feynman in The Character of Physical Law, page 103. Conservation is based upon constancy. Wherever there is symmetry there is a law of conservation and vice versa.

Why have mass (M), length (L), and time (T) been chosen to parametrize the laws of nature? (The emblem of the American Physical Society uses a brass weight, a ruler, and a pendulum, respectively, to symbolize these three fundamental pillars of physics.) In brief, the reason for this is that the use and purpose of energy is to move mass the greatest distance in the shortest amount of time, and all of these $137 \mathrm{~s}$ are the most effective means of accomplishing this goal.

This principle of nature is the basis of George Dantzig's ingenious simplex method which is the economic procedure used worldwide to calculate the best way to move materials (M) with the least amount of labor (L) in the least amount of time (T). Ergonomically and thermodynamically, this is the optimal way to go because it conserves both energy and time
(Planck's constant of the conservation of action, Energy $\mathrm{x}$ Time $=\mathrm{h}=137+$ ). For example, to move an automobile economically we must calculate the amount of energy (gallons of gasoline) needed to transport its mass the most efficiently (speeds that get the best mileage per gallon, e.g., $65 \mathrm{mph}$ ) in the time allotted for the actual trip, or less.

This optimization of results also appears in biology as well as physics, chemistry, and economics. For examples, the proportion of white to grey matter in animal and human brains minimizes communication delay in the neurons; the firing of brain neurons also follows the (Leibnizian) all-or-nothing binary system coordinated by the minimizing principle; the body locomotion of animals optimizes travel by minimizing the use of energy so as to maintain the right ratio between the distance covered and the amount of energy expended (for this reason many animals leap in accordance with the cycloid arc because it minimizes energy with the quickest descent to the ground).

Quite remarkably. the world is made in terms of weight (M), length (L), and time (T). "Faster, easier, cheaper... [d] esigns evolved so that at each subsequent stage of evolution the newer animal forms were able to cover more area for less consumption of useful energy.... At each stage of this story--the rise of the lithosphere, atmosphere, hydrosphere, and biosphere--nature evolved to facilitate the movement of more mass on earth" (Design in Nature, Adrian Bejan and J. Peder Zane, pages 230 and 252, which brings to mind the ancient expression, "music of the spheres" that is also the music of the primes and the natural elements in a hypersphere).

The principle of least work is also behind the golden ratio arrangement of leaves in plant phyllotaxis that maximizes the amount of light and water for the plant to grow. That this same principle should lie hidden inside of the golden proportion may come as a surprise to us. But experiments show that the eye is able to scan and enjoy the size of an object (L) with the greatest ease $(\mathrm{M})$ in the least amount of time (T) when the object is in the Phi proportions. This is the reason why sheets of paper, playing cards, credit cards, windows, doors, and buildings (like the United Nations building), etc., are designed to be as close to, if not exactly the same as, this famous ratio.

Ideally, this is also the reason why paragraphs should be parsed vertically or horizontally as closely as possible to this golden rectangle proportion in papers and books for easier (speed) reading (as attempted in this paper). "Shapes that resemble the golden ratio facilitate the scanning of images and their transmission through vision organs to the brain" (Design in Nature, Adrian Bejan and J. Peder Zane, page 233). The same thing is true of the primes: the mass of primes is moved an infinite distance (infinite descent) in hardly any time (maybe no time) at all. Also, because of his fascination with the golden proportion in nature, Alan Turing used this ratio as the basis of the parsing theorem in digital computers.

That there is such an intimate relationship between mathematics and physics, just as there is between mathematics and music ("architecture is frozen music"), can be seen not only in hydrogen with both $137 \mathrm{~s}$ represented in it but also in 
the equation between light-speed (137+) and the square root of minus one. This equivalence changes one dimension into another (time into space and vice versa), just like the golden proportion, because of its unique combined arithmetical (additive) and geometrical (multiplicative) properties. Just as the two 137s appear in hydrogen, they also appear as the transformation of one dimension (or derivative) into another. Like Phi, a wavicle of light is both a whole and a part. Also, because Phi ( $\phi)$ is reciprocally related to $i$, and $i$ is equivalent to the speed of light, Phi is therefore related to light-speed as well. Remember: according to Einstein's special theory of relativity, $(i \phi)+1 /(i \phi)=i$.

Also, according to Einstein's special theory of relativity, at the speed of light an object becomes a cross-section of itself. If the first dimension is a cross-section of the second, and the second is a cross-section of the third, etc., what is the zero dimension (null line of light)? The answer is that it is its own cross-section, just as Phi is a cross-section of itself. In a word, both are self-contained and self-congruent, like a self-referral fractal. With the addition of light-speed (i), using Planck's natural units, we obtain the stunning equation, Leibniz's "imaginary constant" (i) = Einstein's constant (c) = Planck's constant $(\mathrm{h})=$ Boltzmann's constant $(\mathrm{k})=$ Newton's constant $(\mathrm{G})=$ Coulomb's constant (epsilono ) $=1$ or unification, all in one fell swoop, because e2 (Feynman's constant) $=137+$.

The path of light is the prime direction; it goes with the flow. (Similarly, primes "grow with the flow" according to the natural logarithm.) Early on in his life, Leibniz lavishly praised the square root of minus one $(i)$ as "the portent of the ideal world, almost the mean between something and nothing," one dimension and another, one derivative and another--the (quantum) nothing that is. By rights, this is how the dimensions unfold--in scale. Physicist John Wheeler, who coined the term "geometrodynamics," put it this way, "Physics is really geometry... Some profound connection exists between the fundamental constants of microphysics and the geometry of the cosmos."

He also emphasized that "A percent or so change one way in one of the 'constants,' $h c / 2$ pi $^{2}$ [Planck's quantum mechanical constant of stationary action $=137+$ ], will cause all stars to be red stars; and a comparable change the other way will make all stars be blue stars.... In neither case will any star like the sun be possible.... Man could never come into being in such a universe" (The Anthropic Cosmological Principle, John Barrow and Frank Tipler, page vii). In other words, to put it bluntly, if the number of this constant were somewhat different, you and I would not be here.

When $137+$ is combined with two other fundamental constants, namely, $1 / 1836$, which is the ratio of the electron to proton mass, and Newton's gravitational constant, these three constants are responsible for scaling the entire universe from the smallest sub-atomic particles to the largest super-superclusters of galaxies. Eadem mutata resurgo ("Though transformed, I rise again the same," is how Jacob Bernoulli, a student and friend of Leibniz, described it.)

This multi-dimensional scaling means that the information of the universe remains essentially the same (recursive, symmetrical or congruent) throughout its stratified (scaled) transformations. This is because ratification is stratification according to the constant rate of these golden numbers, as seen in the prime numbers, the layers (spheres) of nature, the periodic table of natural elements, etc.

In a word, the universe, among other things, is recursive.

\section{Taming the Wild Infinite Fibonacci Sequence}

More support for this uni-Phi-cation of mathematics and physics comes from the fact that there is an intimate connection between the rounded up (octave) number 7-14-28-57 and the digital decimal system because of the "rule of nines" or "nine-test" (zero through nine are the basis of the decimal code) of which both Leibniz and Ramanujan were so fond. (Ramanujan used it successfully to partition numbers; he was also fascinated by the mod 24 elliptic function that we have previously used to parse the prime numbers.) The sum of the digits of 142857 equals 9 , and the division of this number in half produces nines: 142 plus 857 equals 999. Also, 142,857 times 7 equals 999,999. Curiously, the "24-carat" golden proportion is also connected to nine through digital summation. The digits of the Fibonacci sequence, like the prime number digital 24-hour clock, recur in a cycle (clock) of 24 (hours):

$$
1,1,2,3,5,8,4,3,7,1,8,9,8,8,7,6,4,1,5,6,2,8,1,9
$$

And then the cycle repeats itself again forever. (Keep going and see.)

When divided in half, with one set of the first twelve numbers superimposed onto and added to the second set of twelve numbers, the sums of the digits all equal nines:

112358437189

887641562819

$999999999999(18$ or 1 plus 8$)$

While intentionally playing around (calculating the variations) with this classical method of compression to digital roots, I found that all non-twin digits after 3 in the prime number 24-hour clock sum up to one of these same six numbers, e.g., 5 is 5,7 is 7,11 is 2,13 is 4,17 is 8,19 is 1,23 is 5,29 is 2,31 is 4,37 is 1 , etc. The primes are digitized.

Moreover, all of the primes are located on elliptic arcs of this modular clock for the reason that the solution to all elliptic equations sum up to these same six numbers. For example, $x^{3}$ $x^{2}=y^{2}+y$ has solutions $L=1, L=4, L=4, L=8, L=16$ (7), etc. All elliptic curves (arcs) in the prime number arcades have an $L$-series that requires prime numbers, and all elliptical equations are modular forms that formulate modular clocks. In the $L$-function is the elliptical function; in the elliptical function is the modular form; and in the modular form is the modulus for prime numbers.

Strangely enough, not only do single primes after 3 sum up to one of the numbers in .142857 , but so do twin primes after 3 
and 5 add up to the numbers 3,6, and 9, which are the remaining three numbers of the decimal system that are missing from the original decimal sequence of .142857 . For examples, $5+7=12=3,11+13=24=6,17+19=36=9$, $29+31=60=6,41+43=84=12=3,59+61=120=3,149$ $+151=300=3,191+193=384=6,227+229=456=6$, $251+253=504=9,257+259=516=3$, etc. If we place zero in the middle of this prime number-clock of one through nine, we have all of the digits necessary for the decimal system, with the number nine beginning each new period in the entire clock.

What happens if we continue this procedure of summing up digits with respect to so-called "cousin primes"--primes separated by four numbers? Not too surprisingly, after 3 and 7 which equal $1(1,3,7$, again), all the others equal 3,6 , and 9: $7+11=9,19+23=6,37+41=6,43+47=9,67+71=3$, $79+83=9,97+101=9,103+107=3,109+113=6,127+$ $131+6,163+167=6,193+197=3$, etc.

And what results if we continue to perform this procedure with regard to "sixy" primes--primes separated by six numbers? Let the reader see for him/herself. (You will be returned to the original six numbers of the clock-block.) Because of the cyclical decimal-based system itself, it is not surprising that all digits sum up to a single decimal digit, but why must single primes add up to one of the numbers in $1 / 7$ or .142857? The answer is that this decimal fraction is the foundation of the decimal/binary code, as previously shown in the table of decimal/binary numbers with the 1, 3, 7, etc., spacing. It is as if successive throws of two loaded dice follow this method of digital summation so that the sums are all digitized (the numbers on the opposite sides of a single die add up to seven).

The connection between prime numbers and the nine-test luckily, however, is a necessary one. A necessary and sufficient condition for any number to be divisible by nine is that its digital sum also be divisible by nine. According to the (prime number) theorem of French mathematician M. E. Midy, if the denominator of a fraction is a prime, like $1 / 7$, and the sum of its period is an even number, each number in the first half of the period together with each digit in the second half adds up to nine, e.g., .142857 splits and becomes $1+8,4+5$, and $2+7$. Moreover, $14+28+57=99$ or 18 or 9 . In other words, the rule of nines helps to decide whether or not a number is prime: if the sum of all digits is a multiple of three or nine, then the number itself is a multiple of three or nine. The nine-test is a prime test. In other words, the block of $1 / 7$ is the clock. The primes are all mode-locked in this mod 24 clock according to the cyclical progression .142857. Mode-clocking is mode-locking; it is the modular clock that makes the prime numbers tick.

Just to top it off, it so happens that in automotive engineering and repair service the rule of nines is used to adjust and synchronize the valves on $\mathrm{V}$-eight engines so that they don't rotate as much as when they are adjusted differently (the calculation of variations leads to this optimal result). Like the eight "hands" of the prime number clock, the eight (!) rocker arms and the eight valves are moving most efficiently (least action, maximum effect) when their settings all precisely follow summations to nine. The sums respectively all equal nines when the first rocker arm has its valve wide open to eight, the third rocker arm has its valve open to six, the fifth rocker arm has its valve open to four, the second arm open to seven, the eighth arm to one, the sixth to three, the fourth to five, and the seventh to two. So, just as the "motor" of the primes is tuned in accord with the nine-test through the fraction $1 / 7$, so are countless vehicle engines "tuned up" in accord with it as well.

Even though Leibniz was very enamored of casting out nines, he did not know about the recurring pattern in Phi, nor did he know that any two numbers, using the Fibonacci rule of addition and division, would converge to Phi. Had he known, he might have compressed his binary code to a unitary one based upon Phi--the true "decimal point" for the decimal ("dicemal"?) system. Just as all of logic is based on a single connective, "nand" that combines "and," "not," and "or" into "not both this and that," all mathematics is based on Phi. In other words, just draw a distinction, a cleft that cleaves to itself and is a "perfect continence," and that is all you need. This single distinction is how G. Spencer-Brown succinctly begins the book Laws of Form in which all the laws of logic can be derived from this special form that sounds a lot like Phi: "We are, and have been all along, deliberating the form of a single construction, notably the first distinction," he writes (on page 68).

\section{Conclusion}

This unification of mathematics through Phi should not come as a complete surprise to us since Phi is related to all three means that are essential to mathematics--the arithmetic, the geometric and the harmonic. (These three means are the result of the calculus of differences, just as the harmonic intervals in music are the result of the calculus of variations.) What does come as a curious surprise is what happens when we begin with the first four numbers of the Fibonacci sequence and then add them together along with their unity to obtain the number seven, plus the appearance of the relationship of the Fibonacci golden angle number to this same number in the binary code, as we have seen.

Also because of Euler's "most remarkable formula," $e^{\pi i}+1$ $=0$, or what might be called the "Midas formula" because of its numerous gold nuggets, this is a truly uni-Phi-ed theory of mathematics: all of the fundamental concepts make their dramatic appearance in it--arithmetic (addition and subtraction of numbers), geometry (pi, multiplication and division), analysis (the natural logarithm and exponential growth), algebra (the square root of minus one), and the initial numbers (zero, negative one and positive one). This unification happily occurs because of seven (!) numbers bunched up at the very beginning of the number system: the square root of minus one, minus one itself, zero, plus one, $1.618 \ldots, 2.718 \ldots, 3.1415 \ldots$, that are further compressible through the binary system down to Phi itself through Fibonacci progressions. 
By means of digital summation all decimal numbers (10) are translated into the binary system $(1,0)$, and by means of the process of collection and division all numbers are convertible into sequences that converge to the golden ratio ( $\phi$, the superposition of 1 and 0 ). This is the most precious jewel in the gold crown of the "Queen of the sciences."

Not by chance does the word "digit" come from our ten fingers: the hands are completely sectioned in accordance with the Fibonacci sequence that asymptotically approaches the golden section number. When we clench the fist, this sequence surprisingly appears in the form of the Fibonacci golden spiral. As we have seen, the digital decimal system shares the same number of digits as the hands do, and the conversion of this code to the simple binary code sequentially follows the progression 1, 3, 7, etc., using the smallest, simplest, most fundamental cyclical number fraction, $1 / 7$.

Given this long string of intrinsic and necessary connections, isn't this famous proportion the elusive pot of gold at the end of the (1, 3, 7 or unitriseptium) rainbow rhapsody of primes and the entire number system?

\section{References}

[1] John Barrow, The Constants, New York, NY: Pantheon, 2002.

[2] Marcus Chown, "Go figure. Why should nature have a favourite number?" New Scientist, Dec. 21/28, 2002.

[3] Radu Coldea, et al., "Quantum Criticality in an Ising Chain: Experimental Evidence for Emergent E8 Symmetry," Science, Jan. 8 2010. ("Public release: Golden ratio discovered in quantum world. Hidden symmetry observed for the first time in solid state matter: http://www.eurekalert.org/pub_releases/ 2010-01/haog-grd0010510.php)"
[4] Tobias Dantzig, Number, The Language of Science, fourth edition, New York, NY: the Free Press, 1954.

[5] Richard Feynman, QED, The Strange Theory of Light and Matter, Princeton, NJ: Princeton University Press, 1985.

[6] Carlos Figueroa, G. Julio Campos, A. Lamberto Castro, "Study of prime numbers through their historical development applying matlab," International Journal of Development Research, 2014, vol. 4, no. 12.

[7] David F. Haight, "Summa Characteristica and the Riemann Hypothesis: scaling Riemann's mountain," the Journal of Interdisciplinary Mathematics, vol. 11, no. 6, Dec. 2008. (Reprinted online by Taylor and Francis Publishing Group, May 2013.)

[8] David F. Haight, "Generalizing Riemann: from the L-Functions to the Birch/Swinnerton-Dyer conjecture," the Journal of Interdisciplinary Mathematics, vol. 13, no. 5, Oct 2010. (Republished online by Taylor and Francis Group, May 2013.)

[9] H. E. Huntley, The Divine Proportion, New York, NY: Dover Publications, Inc., 1970.

[10] Gottfried Wilhelm Leibniz, Monadology and other Philosophical Writings, ed. R. Latta, Oxford: Oxford University press, 1965.

[11] Leibniz, Philosophical Writings, ed. G. H. R. Parkinson, London: Everyman's Library, 1983.

[12] Leibniz, Selections, ed. P. Wiener, New York, NY: Charles Scribner's Sons, 1951.

[13] Roger Penrose, The Road to Reality, New York, NY: Alfred A. Knopf, 2004.

[14] Y. Shang, "A result concerning the ratio of consecutive prime numbers," Mathematical Sciences Letters, 2013, vol. 2, no. 3. 